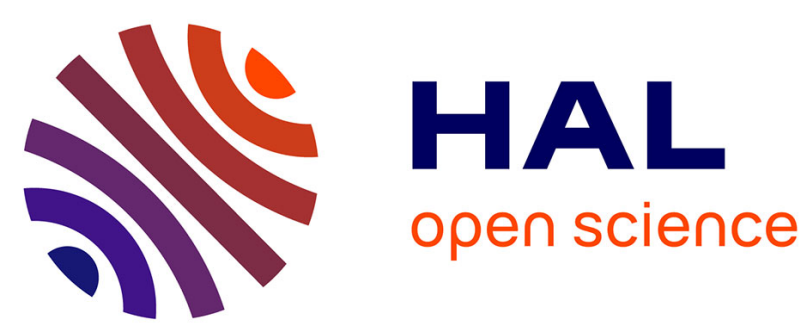

\title{
Construction of a micromechanics-based intralaminar mesomodel, and illustrations in ABAQUS/Standard
}

Gilles Lubineau, Pierre Ladevèze

\section{To cite this version:}

Gilles Lubineau, Pierre Ladevèze. Construction of a micromechanics-based intralaminar mesomodel, and illustrations in ABAQUS/Standard. Computational Materials Science, 2008, 43 (1), pp.137-145. 10.1016/j.commatsci.2007.07.050 . hal-01648329

\section{HAL Id: hal-01648329 \\ https://hal.science/hal-01648329}

Submitted on 18 Dec 2019

HAL is a multi-disciplinary open access archive for the deposit and dissemination of scientific research documents, whether they are published or not. The documents may come from teaching and research institutions in France or abroad, or from public or private research centers.
L'archive ouverte pluridisciplinaire HAL, est destinée au dépôt et à la diffusion de documents scientifiques de niveau recherche, publiés ou non, émanant des établissements d'enseignement et de recherche français ou étrangers, des laboratoires publics ou privés. 


\title{
Construction of a micromechanics-based intralaminar mesomodel, and illustrations in ABAQUS/Standard
}

\author{
G. Lubineau $^{\mathrm{a}, *}, \mathrm{P}$. Ladevèze ${ }^{\mathrm{a}, \mathrm{b}}$ \\ ${ }^{\text {a }}$ LMT-Cachan (E.N.S. de Cachan, Université Paris 6, C.N.R.S., UniverSud Paris), 61 Avenue du Président Wilson, 94235 Cachan Cedex, France \\ ${ }^{\mathrm{b}}$ EADS Foundation Chair, Advanced Computational Structural Mechanics, France
}

\begin{abstract}
The recent advances in the modeling of degradations in stratified composites have led to improved models on all scales. In particular, today, micromechanics derived in a generic framework enables one to define a reference virtual material which integrates most of the knowledge of a material. Thus, a model using damage mechanics on the mesoscale and usable for structural analysis can be built as a homogenized version of this reference model through previously-developed bridges. The objective is to derive a refined model worthy of micromechanics confidence, but transposable into a commercial code (here, ABAQUS/Standard).
\end{abstract}

Keywords: Damage; Laminates; Homogenization

\section{Introduction}

In the past thirty years or so, due to the increasing use of composite materials, the prediction of their degradation has spawned numerous works among diverse communities. Today, degradation models exist on very different scales (from the fiber's scale to the structure's scale, including what is known as the "meso" scale of the elementary layer) using equally varied theoretical tools (fracture mechanics, damage mechanics).

Micromechanics-based models usually address specific mechanisms (primarily transverse cracking [1-5]) under particular loading conditions. The advantage of these models is that they have a strong physical meaning, but they hardly lend themselves to analysis or reanalysis of actual industrial structures.

Another approach developed more recently [6,7] and usable in structural analysis consists in using damage mechanics on the mesoscale. The governing assumption is that the behavior of any stratified structure can be described through two families of basic damageable con-

\footnotetext{
${ }^{*}$ Corresponding author. Tel.: +331474022 36; fax: +33147402785.

E-mail address: lubineau@Imt.ens-cachan.fr (G. Lubineau).
}

stituents: the elementary layer [8] and the interlaminar interface [9]. The behavior of each mesoconstituent is assumed to be intrinsic. This approach provides a general formalism and can be transposed more easily into commercial calculation tools. However, it relies mainly on heuristic laws and a single damage variable is usually associated with several degradation mechanisms, which reduces one's confidence and impairs the readability of the results compared to experimental data.

Continuing the works done in $[10,11]$, in order to bring these two approaches together, a micromechanics reference model was developed $[12,13]$. This micromechanics model is hybrid in the sense that the description of the degradation uses damage mechanics and fracture mechanics alternatively, depending on the mechanism being considered. Thus, diffuse degradation (fiber/matrix splitting) in the elementary layer is described through damage mechanics on the mesoscale according to [8]. Conversely, transverse cracks and associated delamination are described in a discrete manner by introducing minimum surfaces whose rupture is determined by a criterion of the "finite energy recovery rate" type [14]. This micromechanics model plays the role of a virtual reference material. It contains all the material information in the description specific to each 
mechanism. However, it leads to prohibitive numerical costs and requires the use of sophisticated multiscale strategies even for the analysis of geometric details.

The objective of this paper is to present the derivation of a model written according to the damage mechanics formalism, but resulting from the systematic homogenization of this reference model.

In the first section of the paper, the main features of the micromechanics reference model and the degradation mechanisms being considered are reviewed. The rest of the paper concerns the construction of the associated intralaminar model alone.

In the second section, each intralaminar degradation mechanism is introduced progressively into the expression of the strain energy on the mesoscale. While for some mechanisms (rupture along the fibers and diffuse damage) the formulations presented are similar to those used classically, the introduction of transverse cracking relies on the micro-meso relations introduced in $[10,15]$.

The third section compiles the constitutive relations used for each mechanism. In general terms, the meso damage law for transverse cracking is the homogenized version of the micromechanics law.

Finally, the fourth section gives an illustration of the type of result which can be expected with such an approach. Rewriting the model using a damage mechanics formalism on the mesoscale enabled us to introduce it into a commercial analysis code (specifically ABAQUS/Standard), the first results of which are presented. We show that the advantage of this approach is not only that it relies as much as possible on the micro laws, but also that at the end of the calculation it provides for each mechanism separate indicators (microcracking rate in particular) which can be easily interpreted in relation to experiments.

\section{The computational hybrid micromodel for laminates}

Our reference material model is the computational damage micromodel for laminates introduced in $[16,12]$ and described in detail in [13]. The novelty of this approach is that it provides a micromechanics framework within which each classical degradation mechanism is described explicitly by its own optimum model. In this sense, it is completely different from classical micromechanics approaches (see Refs. [17,18] for a review) or models based on damage mechanics (in which the damage variables used represent several underlying mechanisms in a global manner).

Let us first recall that this approach assumes that any complex degradation state can be described as the result of the concurrent development of classically established elementary mechanisms for the material being considered. In the special case of stratified organic composites, these elementary mechanisms are:

- Diffuse intralaminar degradation: this mechanism is associated with degradations on a very small scale (the fiber's scale), such as fiber-matrix splitting or matrix rupture between fibers ([19-21]). This degradation is assumed to be locally homogeneous (on the scale of the elementary ply).

- Transverse intralaminar degradation: this mechanism corresponds to the percolation of diffuse damage into cracks parallel to the fibers which extend completely across the elementary ply. Many studies of this phenomenon can be found in the literature on the micromechanics of stratified materials.

- Diffuse interface degradation: this mechanism concerns microcracks in the interlaminar matrix layer.

- Distributed delamination: transverse microcracks extend throughout the thickness of the elementary ply and are stopped by the interlaminar interface. Thus, they create high-stress zones at the crack tips, leading to local degradations of the interlaminar interface which can be easily observed on the elementary ply's scale $[2,22]$.

- Fiber breakage, which can occur in traction as well as in compression.

The hybrid model is based on the separation of these basic mechanisms, in terms of both scale and morphology, in order to derive the most appropriate model for each mechanism. Our reference scale for studying stratified composites is the mesoscale (i.e. the scale of the elementary ply) because the stacking of these plies is responsible, for the most part, for the heterogeneity of the stress and strain fields in the structure. On this scale, diffuse mechanisms can be viewed as naturally homogenized because they occur on a much smaller scale. Therefore, they are described through damage mechanics in the classical sense of the "damage mesomodel for stratified materials" introduced in [8]. The degradation morphology of discrete mechanisms (transverse cracking and distributed delamination), however, takes place on a scale comparable to the mesoscale. In the hybrid model, we choose to model these degradations in a fully discrete manner through a generalization of the "finite fracture mechanics" concept introduced by [14]. Both transverse cracking and distributed delamination propagate within the structure through complete breakage of "elementary rupture surfaces", the breakage of each interface being controlled by a criterion of the "discrete energy recovery rate" type.

This approach has the advantage of being extremely robust thanks to the discrete vision of the degradation. Besides, it enables the successful calculation, with no major additional assumption, of zones with high solicitation gradients in which complex coupling among the mechanisms (in particular between discrete degradations of the ply and interface) takes place. However, it requires a refined discretization of the structure (several billion DOFs for the analysis of a geometric defect of limited extent), which demands highly effective multiscale strategies [23], and the description retained for the degradation (hybrid continuous/discrete) requires the development of a dedicated calculation tool. 
Therefore, the objective of the next section, on the basis of this micromechanics reference model, is to build a "pragmatic" model compatible with the standard formalisms used in industrial analysis codes, which are often those used in damage mechanics. The resulting model must combine a certain flexibility in the analysis, due to its "meso" aspect, with a certain reliability (contrary to standard damage models) due to its underlying micromechanics bases.

Here, we will limit ourselves to the definition of the associated intralaminar model because the ply can be affected by three purely intralaminar basic mechanisms: fiber breakage, diffuse intralaminar degradation and transverse cracking. The extension to the other mechanisms and to the interface can be completed in order to achieve a nonlocal model coupling intra and interlaminar damage. The details of this more elaborate model have already been mentioned in [24] and its complete development will be the subject of future works.

\section{Strain energy of the damaged ply}

In the elementary ply (i.e. the set of the adjacent fibers with the same direction, which can possibly be composed of several adjacent plies with the same direction), 1, 2 and 3 will denote respectively the direction of the fibers, the transverse direction in the plane of the stratified material and the out-of-plane transverse direction. The material quantities denoted $\left(.^{0}\right)$ refer to the healthy material (exempt from any degradation mechanism).

\subsection{Layer with damage along the fibers alone}

Let us first consider the case of the layer (initially assumed to be transverse isotropic) being possibly degraded by rupture along the fibers quantified by the damage indicator $d_{\mathrm{F}}$. In this case, the layer's strain energy is given by

$$
\begin{aligned}
2 e_{d}= & {\left[\begin{array}{l}
\sigma_{11} \\
\sigma_{22} \\
\sigma_{33}
\end{array}\right]\left[\begin{array}{ccc}
\frac{1}{E_{1}^{0} \cdot\left(1-d_{\mathrm{F}}\right)} & -\frac{v_{12}^{0}}{E_{1}^{0} \cdot\left(1-d_{\mathrm{F}}\right)} & -\frac{v_{12}^{0}}{E_{1}^{0} \cdot\left(1-d_{\mathrm{F}}\right)} \\
-\frac{v_{12}^{0}}{E_{1}^{0} \cdot\left(1-d_{\mathrm{F}}\right)} & \frac{1}{E_{2}^{0}} & -\frac{v_{23}^{0}}{E_{2}^{0}} \\
-\frac{v_{12}^{0}}{E_{1}^{0} \cdot\left(1-d_{\mathrm{F}}\right)} & -\frac{v_{23}^{0}}{E_{2}^{0}} & \frac{1}{E_{2}^{0}}
\end{array}\right]\left[\begin{array}{l}
\sigma_{11} \\
\sigma_{22} \\
\sigma_{33}
\end{array}\right] } \\
& +\frac{\sigma_{12}^{2}}{G_{12}^{0}}+\frac{\sigma_{13}^{2}}{G_{12}^{0}}+\frac{\sigma_{23}^{2}}{G_{23}^{0}}
\end{aligned}
$$

Remark. In order to simplify the expressions, we omitted elastic nonlinearities. These can be introduced easily and are necessary for an accurate representation of the behavior in traction as well as in compression in the fibers' direction.

Remark. As classical in damage mechanics, the range for the damage parameter is limited by the positive definition of the stiffness operator (and not systematically between 0 and 1).

\subsection{Layer with diffuse damage alone}

Here, the layer is assumed to be affected by diffuse intralaminar degradation alone.

The diffuse degradation level of the fiber/matrix material is quantified, as in the damage mesomodel for stratified materials introduced by [8], by two damage variables: $\tilde{d}$ corresponding to the shear moduli and $\tilde{d}^{\prime}$ corresponding to the transverse moduli.

In order to extend the method to three-dimensional calculations, a choice must be made concerning the out-ofplane shape of this part of the model. Our pragmatic choice here consists in assuming that diffuse damage is roughly isotropic.

$$
\begin{aligned}
2 e_{d}= & {\left[\begin{array}{c}
\sigma_{11} \\
\sigma_{22} \\
\sigma_{33}
\end{array}\right]^{\mathrm{t}}\left[\begin{array}{ccc}
\frac{1}{E_{1}^{0}} & -\frac{v_{12}^{0}}{E_{1}^{0}} & -\frac{v_{12}^{0}}{E_{1}^{0}} \\
-\frac{v_{12}^{0}}{E_{1}^{0}} & \frac{1}{E_{2}^{0 \cdot} \cdot\left(1-\left[\sigma_{22}\right]^{+} \tilde{d}^{\prime}\right)} & -\frac{v_{23}^{0}}{E_{2}^{0}} \\
-\frac{v_{12}^{0}}{E_{1}^{0}} & -\frac{v_{23}^{0}}{E_{2}^{0}} & \frac{1}{E_{2}^{0 \cdot} \cdot\left(1-\left[\sigma_{33}\right]^{+} \tilde{d}^{\prime}\right)}
\end{array}\right]\left[\begin{array}{c}
\sigma_{11} \\
\sigma_{22} \\
\sigma_{33}
\end{array}\right] } \\
& +\frac{\sigma_{12}^{2}}{G_{12}^{0}(1-\tilde{d})}+\frac{\sigma_{13}^{2}}{G_{12}^{0}(1-\tilde{d})}+\frac{\sigma_{23}^{2}}{G_{23}^{0}\left(1-\tilde{d}_{23}\right)},
\end{aligned}
$$

where $[x]^{+}=1$ if $x \geqslant 0$ and $[x]^{+}=0$ if $x<0$ in order to introduce the unilateral character of damage.

Remark. The transverse isotropy assumption is an approximation which could be refined through more precise micromechanics studies on the fiber's scale [25]. As it stands, this assumption enables one to retain a simple, common-sense model, given that for the most part the outof-plane behavior is not governed by this mechanism but by delamination possibly coupled with transverse cracking. Thus, the damage $\tilde{d}_{23}$ is not an additional variable, but is defined through transverse isotropy as

$$
\left(1-\tilde{d}_{23}\right)=\frac{1-\tilde{d}^{\prime}}{1-\frac{v_{23}^{0}}{1+v_{23}^{0}} \tilde{d}^{\prime}}
$$

Remark. In the classical version of the damage mesomodel for stratified materials, the two damage variables $\tilde{d}$ and $\tilde{d}^{\prime}$ represent globally the modulus reductions due to the different mechanisms. Here, only the diffuse mechanism is concerned by these two variables.

\subsection{Layer with transverse cracking alone}

Here, we assume that the layer is affected only by transverse cracking.

Assuming that transverse cracking is locally periodical and quantified by the classically associated micromechanics variable $\rho$ (microcracking rate), the micro-meso relations developed previously in $[10,11]$ enable one to homogenize this mechanism in order to define three damage indicators $\bar{d}_{12}, \bar{d}_{22}$ and $\bar{d}_{23}$. These indicators are not independent 
because they are all connected to the microcracking rate $\rho$ through the layer homogenization problem.

In this context, the strain energy density of the degraded layer is given by Eq. (4). (The calculations leading to this equation are detailed in Annex A.)

$$
\begin{aligned}
2 e_{d}= & {\left[\begin{array}{l}
\sigma_{11} \\
\sigma_{22} \\
\sigma_{33}
\end{array}\right]^{\mathrm{t}}\left[\begin{array}{ccc}
\frac{1}{E_{1}^{0}} & -\frac{v_{12}^{0}}{E_{1}^{0}} & -\frac{v_{12}^{0}}{E_{1}^{0}} \\
-\frac{v_{12}^{0}}{E_{1}^{0}} & \frac{1}{\left(1-\left[\sigma_{22}\right]^{+} \bar{d}_{22}\right) E_{2}^{0}} & -\frac{v_{23}^{0}}{\left(1-\left[\sigma_{22}\right]^{+} \overline{2}_{22}\right) E_{2}^{0}} \\
-\frac{v_{12}^{0}}{E_{1}^{0}} & -\frac{v_{23}^{0}}{\left(1-\left[\sigma_{22}\right]^{+} \bar{d}_{22}\right) E_{2}^{0}} & \frac{1}{E_{2}^{0}}
\end{array}\right]\left[\begin{array}{l}
\sigma_{11} \\
\sigma_{22} \\
\sigma_{33}
\end{array}\right] } \\
& +\frac{\sigma_{12}^{2}}{G_{12}^{0}\left(1-\bar{d}_{12}\right)}+\frac{\sigma_{13}^{2}}{G_{12}^{0}}+\frac{\sigma_{23}^{2}}{G_{23}^{0}\left(1-\bar{d}_{23}\right)}
\end{aligned}
$$

Remark. Here, the advantage of constructing the mesomodel through the homogenization of micromechanics schemes is obvious because this approach governs the form of the energy for each mechanism. It is clear that transverse cracking affects only components 22,12 and 23, and that the effect of transverse cracking in each of these directions is known and imposed by micromechanics.

\subsection{Degraded layer in the general case}

Now, let us assume that the layer is affected by the three previous mechanisms simultaneously. Then, the resulting strain energy density is given by

$$
\begin{aligned}
2 e_{d}= & {\left[\begin{array}{l}
\sigma_{11} \\
\sigma_{22} \\
\sigma_{33}
\end{array}\right]^{\mathrm{t}}[S]\left[\begin{array}{l}
\sigma_{11} \\
\sigma_{22} \\
\sigma_{33}
\end{array}\right]+\frac{\sigma_{12}^{2}}{G_{12}^{0}\left(1-\bar{d}_{12}\right)(1-\tilde{d})}+\frac{\sigma_{13}^{2}}{G_{13}^{0}(1-\tilde{d})} } \\
& +\frac{\sigma_{23}^{2}}{G_{23}^{0}\left(1-\bar{d}_{23}\right)\left(1-\tilde{d}_{23}\right)},
\end{aligned}
$$

where $[S]$ is defined by

$$
\left[\begin{array}{ccc}
\frac{1}{E_{1}^{0}\left(1-d_{\mathrm{F}}\right)} & -\frac{v_{12}^{0}}{E_{1}^{0}\left(1-d_{\mathrm{F}}\right)} & -\frac{v_{12}^{0}}{E_{1}^{0}\left(1-d_{\mathrm{F}}\right)} \\
-\frac{v_{12}^{0}}{E_{1}^{0}\left(1-d_{\mathrm{F}}\right)} & \frac{1}{\left(1-\left[\sigma_{22}\right]^{+} \bar{d}_{22}\right)\left(1-\left[\sigma_{22}\right]^{+} \tilde{d}^{\prime}\right) E_{2}^{0}} & -\frac{v_{23}^{0}}{\left(1-\left[\sigma_{22}\right]^{+} \bar{d}_{22}\right) E_{2}^{0}} \\
-\frac{v_{12}^{0}}{E_{1}^{0}\left(1-d_{\mathrm{F}}\right)} & -\frac{v_{23}^{0}}{\left(1-\left[\sigma_{22}\right]^{+} \bar{d}_{22}\right) E_{2}^{0}} & \frac{1}{E_{2}^{0}\left(1-\left[\sigma_{33}\right]^{+} \tilde{d}^{\prime}\right)}
\end{array}\right]
$$

Remark. Eq. (5) assumes that on the scale of the cracking step the diffuse degradation remains locally homogeneous in spite of the existence of transverse cracks. One could argue about this assumption, but Eq. (5) introduces between these two mechanisms a "small" coupling which is probably sufficient in most cases.

Remark. These degradation mechanisms (particularly diffuse degradation) are accompanied by unrecoverable plastic behavior due, in our case, to friction along the splitting interfaces. The classical model introduced in [8] can be used provided, of course, that the effective stresses are redefined according to the modified damage equation.

\section{Constitutive relations}

Having described the different mechanisms separately on the strain energy level (Expression (5)), we can now develop each mechanism using separate constitutive relations as well.

\subsection{Rupture along the fibers/diffuse degradation}

These mechanisms are classically introduced into the damage mesomodel for stratified materials. Therefore, we can reuse the classical constitutive relations defined in this framework. Let us simply observe that even though the definition of the associated thermodynamic forces remains unchanged from the classical model, their definition takes the existence of transverse cracking into account through Expression (5).

Thus, concerning fiber breakage, we retain a brittle law which distinguishes rupture in traction and rupture in compression through two thresholds $Y_{\mathrm{F}}^{\mathrm{t}}$ and $Y_{\mathrm{F}}^{\mathrm{c}}(\langle\langle\cdot\rangle\rangle$ representing the mean value across the layer).

$$
Y_{d_{\mathrm{F}}}=-\frac{\partial\left\langle\left\langle e_{d}\right\rangle\right\rangle}{\partial d_{\mathrm{F}}}
$$

- while $Y_{d_{\mathrm{F}}}<Y_{\mathrm{F}}^{\mathrm{t}}$ and $Y_{d_{\mathrm{F}}}<Y_{\mathrm{F}}^{\mathrm{c}} d_{\mathrm{F}}=0$

- if $Y_{d_{\mathrm{F}}}>Y_{\mathrm{F}}^{\mathrm{t}}$ and $\sigma_{11}>0$, then $d_{\mathrm{F}}=1$ (rupture in traction)

- if $Y_{d_{\mathrm{F}}}>Y_{\mathrm{F}}^{\mathrm{c}}$ and $\sigma_{11}<0$, then $d_{\mathrm{F}}=1$ (rupture in compression)

Regarding diffuse damage, its progressive evolution is given by the classical law

$$
\begin{array}{ll}
Y_{\tilde{d}}=-\frac{\partial\left\langle\left\langle e_{d}\right\rangle\right\rangle}{\partial \tilde{d}} ; & Y_{\tilde{d}^{\prime}}=-\frac{\partial\left\langle\left\langle e_{d}\right\rangle\right\rangle}{\partial \tilde{d}^{\prime}} \\
\tilde{Y}=Y_{\tilde{d}}+b_{2} Y_{\tilde{d}^{\prime}} ; \quad \tilde{d}=\sup _{\tau \leqslant t} \frac{\sqrt{Y}-\sqrt{Y_{0}}}{\sqrt{Y_{\mathrm{c}}}-\sqrt{Y_{0}}} ; \quad \tilde{d}^{\prime}=b_{3} \tilde{d}
\end{array}
$$

$b_{2}, b_{3}, Y_{0}$ and $Y_{\mathrm{c}}$ are experimentally identified quantities. Let us note that the progressive part of the constitutive relation alone is preserved. The thresholds initially introduced in [8] were intended to represent the rapid development of the microcracks, which is now described separately in the following section. 


\subsection{Evolution of transverse cracking}

Now, let us derive the constitutive relation for transverse cracking in the elementary ply using a micromechanics model with the following assumptions:

- The evolution of cracking is governed by a criterion of the "energy recovery rate" type. We choose a continuous representation as a function of $\rho$ :

$$
G=-\frac{\partial E_{\mathrm{p}}}{\partial A},
$$

where $E_{\mathrm{p}}$ is the potential energy of the degraded layer and $A$ is the area of the transverse cracks. It was shown in [13] that, by taking into account the variabilities, this definition leads to a good approximation of the discrete energy recovery rate $\left(\Delta G=-\Delta E_{\mathrm{p}} / \Delta A\right)$ used in micromechanics.

- Transverse cracking, as observed experimentally (e.g. [26]) is assumed to stop once a limit microcracking rate $\rho_{s}$ has been reached. Beyond this limit, interlaminar phenomena take place (especially distributed delamination), which requires more elaborate models.

- Here, we are developing only the criterion for "thin" layers (at most two or three elementary plies, which is true of most industrial cases). Thus, the micromechanics model is based entirely on an energy criterion. More complex models including the behavior of thick plies [13] could be introduced if necessary.

Let us consider the integral of the energy density over a length $L$ of the elementary ply of thickness $H$. Let $A$ be the cracked area within this zone. Thus, starting from (5) and (10), the energy recovery rate, which plays the role of the damage force associated with the evolution of the transverse cracks, can be expressed as $(\langle\cdot\rangle$ representing the positive part)

$$
\begin{aligned}
\bar{Y}_{\rho}= & \frac{\partial E_{d}}{\partial A} \\
= & L H\left[\frac{\left\langle\sigma_{22}\right\rangle_{+}}{2 E_{2}^{0}\left(1-\bar{d}_{22}\right)^{2}\left(1-\tilde{d}^{\prime}\right)}-\frac{v_{23}^{0}}{E_{2}^{0}\left(1-\bar{d}_{22}\right)^{2}} \sigma_{33}\left\langle\sigma_{22}\right\rangle_{+}\right] \frac{\partial \bar{d}_{22}}{\partial A} \\
& +L H\left[\frac{\sigma_{12}^{2}}{2 G_{12}^{0}\left(1-\bar{d}_{12}\right)^{2}(1-\tilde{d})}\right] \frac{\partial \bar{d}_{12}}{\partial A} \\
& +L H\left[\frac{\sigma_{23}^{2}}{2 G_{23}^{0}\left(1-\bar{d}_{23}\right)^{2}\left(1-\tilde{d}_{23}\right)}\right] \frac{\partial \bar{d}_{23}}{\partial A}
\end{aligned}
$$

Now, let us introduce the notations

$$
\begin{aligned}
& \bar{Y}_{22}=H\left[\frac{\left\langle\sigma_{22}\right\rangle_{+}}{2 E_{2}^{0}\left(1-\bar{d}_{22}\right)^{2}\left(1-\tilde{d}^{\prime}\right)}-\frac{v_{23}^{0}}{E_{2}^{0}\left(1-\bar{d}_{22}\right)^{2}} \sigma_{33}\left\langle\sigma_{22}\right\rangle_{+}\right] \\
& \bar{Y}_{12}=H\left[\frac{\sigma_{12}^{2}}{2 G_{12}^{0}\left(1-\bar{d}_{12}\right)^{2}(1-\tilde{d})}\right]
\end{aligned}
$$

$\bar{Y}_{23}=H\left[\frac{\sigma_{23}^{2}}{2 G_{23}^{0}\left(1-\bar{d}_{23}\right)^{2}\left(1-\tilde{d}_{23}\right)}\right]$

$\bar{Y}_{22}, \bar{Y}_{12}$ and $\bar{Y}_{23}$ are not strictly damage forces because the variables $\bar{d}_{22}, \bar{d}_{12}$ and $\bar{d}_{23}$ are not independent, but related to the underlying quantity $\rho$. They are distributions of the damage force $\bar{Y}_{\rho}$ according to the modes.

Then, considering that $\rho=A / L$, Expression (11) can be rewritten as

$\bar{Y}_{\rho}=\left[\bar{Y}_{22} \frac{\partial \bar{d}_{22}}{\partial \rho}+\bar{Y}_{12} \frac{\partial \bar{d}_{12}}{\partial \rho}+\bar{Y}_{23} \frac{\partial \bar{d}_{23}}{\partial \rho}\right]$,

where $G_{\mathrm{I}}^{\mathrm{c}}, G_{\mathrm{II}}^{\mathrm{c}}$ and $G_{\mathrm{III}}^{\mathrm{c}}$ designate the critical energy recovery rates in Mode-I, Mode-II and Mode-III, respectively. The rupture envelope is then described by a classical mixed criterion of fracture mechanics

$$
\left[\left(\frac{\bar{Y}_{22} \frac{\partial \bar{d}_{22}}{\partial \rho}}{G_{\mathrm{I}}^{\mathrm{c}}}\right)^{\alpha}+\left(\frac{\bar{Y}_{12} \frac{\partial \bar{d}_{12}}{\partial \rho}}{G_{\mathrm{II}}^{\mathrm{c}}}\right)^{\alpha}+\left(\frac{\bar{Y}_{23} \frac{\partial \bar{d}_{23}}{\partial \rho}}{G_{\mathrm{III}}^{\mathrm{c}}}\right)^{\alpha}\right]^{\frac{1}{\alpha}}=1
$$

Finally, this leads to the following evolution of microcracking:

- if $\rho \leqslant \rho_{s}$

$$
\begin{aligned}
& \rho=\sup _{\tau \leqslant t}\left[\mathscr{G}\left\{\left(\left(\gamma_{\mathrm{I}} \bar{Y}_{22}\right)^{\alpha}+\left(\gamma_{\mathrm{II}} \bar{Y}_{12}\right)^{\alpha}+\left(\gamma_{\mathrm{III}} \bar{Y}_{23}\right)^{\alpha}\right)^{\frac{1}{\alpha}}\right\}\right] \\
& \bar{d}_{22}=f_{22}(\rho), \quad \bar{d}_{12}=f_{12}(\rho), \quad \bar{d}_{23}=f_{23}(\rho) \\
& \gamma_{\mathrm{I}}=\frac{1}{G_{\mathrm{I}}^{\mathrm{c}}}, \quad \gamma_{\mathrm{II}}=\frac{\frac{\partial f_{12}}{\partial \rho}}{\frac{\partial f_{22}}{\partial \rho} G_{\mathrm{II}}^{\mathrm{c}}}, \quad \gamma_{\mathrm{III}}=\frac{\frac{\partial f_{23}}{\partial \rho}}{\frac{\partial f_{22}}{\partial \rho} G_{\mathrm{III}}^{\mathrm{c}}} \\
& \mathscr{G}: x \rightarrow \frac{\partial f_{22}{ }^{-1}}{\partial \rho}\left(\frac{1}{x}\right)
\end{aligned}
$$

- otherwise

$$
\rho=\rho_{s} ; \quad \bar{d}_{22}=f_{22}\left(\rho_{s}\right) ; \quad \bar{d}_{12}=f_{12}\left(\rho_{s}\right) ; \quad \bar{d}_{23}=f_{23}\left(\rho_{s}\right)
$$

Functions $\quad\left[\bar{d}_{22} \rightarrow \rho\right], \quad\left[\bar{d}_{12} \rightarrow \rho\right] \quad$ and $\quad\left[\bar{d}_{23} \rightarrow \rho\right] \quad$ are obtained through the homogenization of the layer problem which defines the micro-meso relations as presented in [10].

\section{First example of application}

\subsection{Implementation}

The previous section enabled us to build a model which follows the formalism of damage mechanics on the mesoscale, yet includes (and is based on) the micromechanics contained in a micromechanics reference model. This formalism can be implemented into a commercial analysis code. Such codes usually follow an incremental strategy 
with respect to time for the resolution of nonlinear problems, and the implementation of our model consists in creating a new material behavior within what is called the "local" loop.

With this technique, our model was implemented into ABAQUS/Standard, which enables one to create a new material behavior through the user subroutine U-MAT. The only difficulty at this point is related to the management of the nonlocal aspect of the model, which defines the damage forces using integrals or mean values over the thickness of the elementary layer. This requires that when the behavior at each integration point is being calculated information about its vicinity be available, which is not the case a priori in the U-MAT formalism. Solutions to make this feature available in the case of arbitrary discretizations are being sought. In the example presented in (5.2), this point was treated pragmatically through the use of a linear element subintegrated across the thickness.

The four sources of intralaminar nonlinearities mentioned in Section 4 were implemented: damage by fiber rupture, diffuse damage, transverse cracking damage and plasticity. Each nonlinearity is solved successively, plasticity being dealt with after damage using a radial recovery algorithm.

Remark. The damage laws which we introduced classically lead to localization and mesh dependency phenomena unless special precautions are taken. We used a formalism with delay effect for all these laws in order to limit this localization $[27,28]$.

\subsection{Illustration}

For our first example, let us consider the case of the holed plate $\left[0_{2} / 90_{2}\right]_{\mathrm{s}}$ shown in Fig. 1 . This problem was inspired by the availability of detailed experimental results in [29]. The calculation was performed in traction with pre-
Table 1

Material properties used for elasticity and damage laws

\begin{tabular}{llll}
\hline$E_{1}^{0}(\mathrm{MPa})$ & $E_{2}^{0}(\mathrm{MPa})$ & $v_{12}^{0}$ & $v_{23}^{0}$ \\
\hline 148,000 & 9570 & 0.356 & 0.49 \\
$G_{12}^{0}(\mathrm{MPa})$ & $b_{2}$ & $b_{3}$ & $Y_{\mathrm{c}}(\mathrm{MPa})$ \\
4500 & 1 & 1 & 15 \\
$Y_{0}(\mathrm{MPa})$ & $Y_{\mathrm{F}}^{\mathrm{t}}(\mathrm{MPa})$ & $Y_{\mathrm{F}}^{\mathrm{c}}(\mathrm{MPa})$ & $\alpha$ \\
0.02 & 10 & 10 & 1 \\
$\rho_{s}$ & $G_{\mathrm{I}}^{\mathrm{c}}\left(\mathrm{J} / \mathrm{m}^{2}\right)$ & $G_{\mathrm{II}}^{\mathrm{c}}\left(\mathrm{J} / \mathrm{m}^{2}\right)$ & $G_{\mathrm{III}}^{\mathrm{c}}\left(\mathrm{J} / \mathrm{m}^{2}\right)$ \\
0.7 & 100 & 300 & 300 \\
\hline
\end{tabular}

scribed displacements and limited to one-quarter of the geometry due to symmetry considerations.

The material quantities used in the calculation are defined in Table 1. They correspond to standard quantities for a carbon-epoxy material, but do not relate to a specific material.

Figs. 2 and 3 show the maps of the different types of damage in the two types of plies for a $0.633 \%$ longitudinal deformation of the plate, near final rupture. At higher loading levels, a delamination mechanism along with coupling between splitting and microcracking appeared clearly and would require the use of more elaborate models with intra-interlaminar coupling [24].

The $90^{\circ}$ ply was free of fiber rupture. Slight diffuse damage progressed throughout the plate. The main point to be observed is the development of a relatively high microcracking level in an extensive zone going from the hole to the edge of the sample, limited by splitting in the $0^{\circ}$ ply.

The $0^{\circ}$ ply was significantly degraded by transverse cracking in the longitudinal direction of the sample and tangent to the hole, which represents the splitting observed experimentally. Initial fiber breakage also started to appear at the edge of the hole.

These results are perfectly consistent with those obtained with X-ray radiography on this type of laminates and presented in [29]. The interest of this type of simulation is that it provides not only a local damage level, but also a

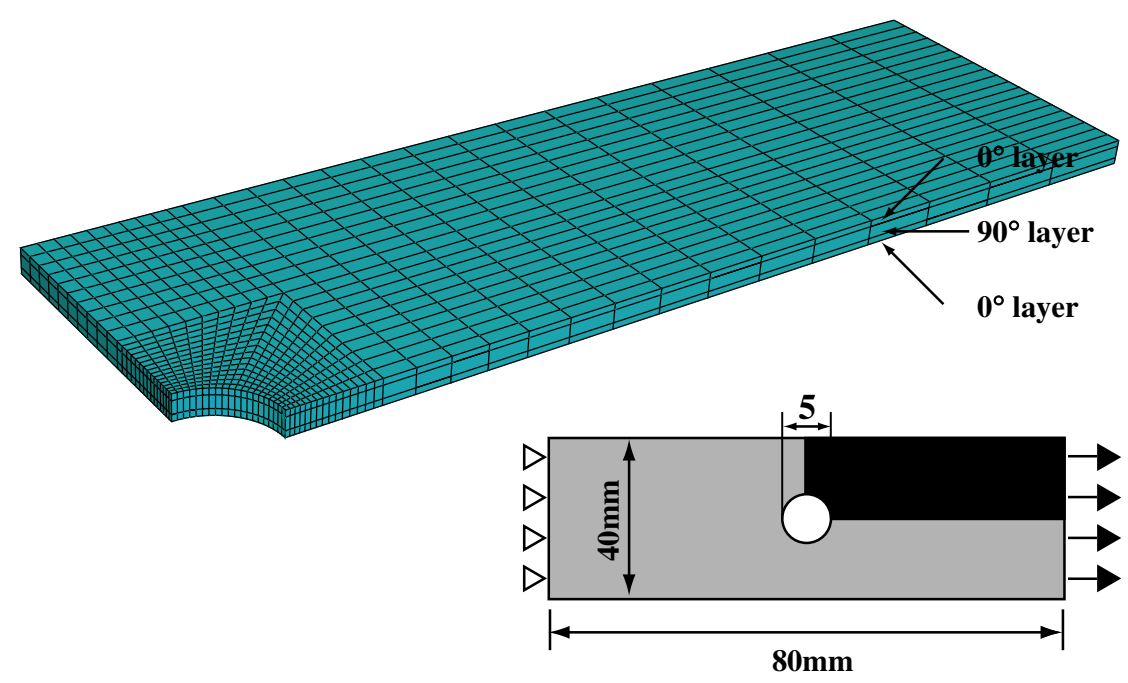

Fig. 1. Finite element model of the $\left[0_{2} / 90_{2}\right]_{s}$ plate. 

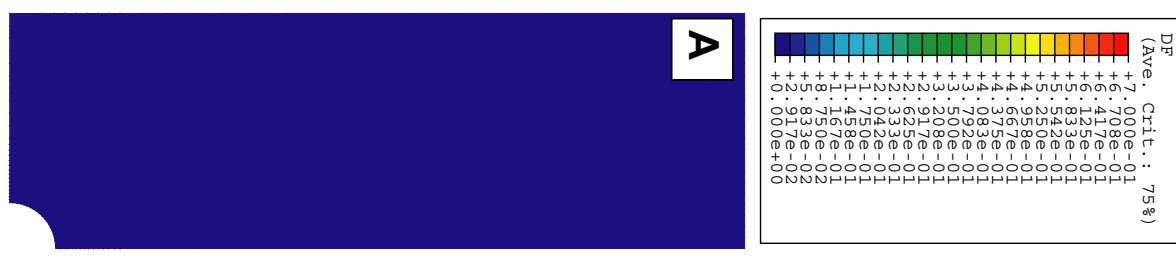

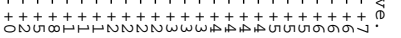

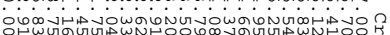

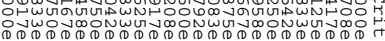

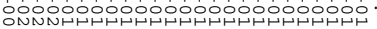

un
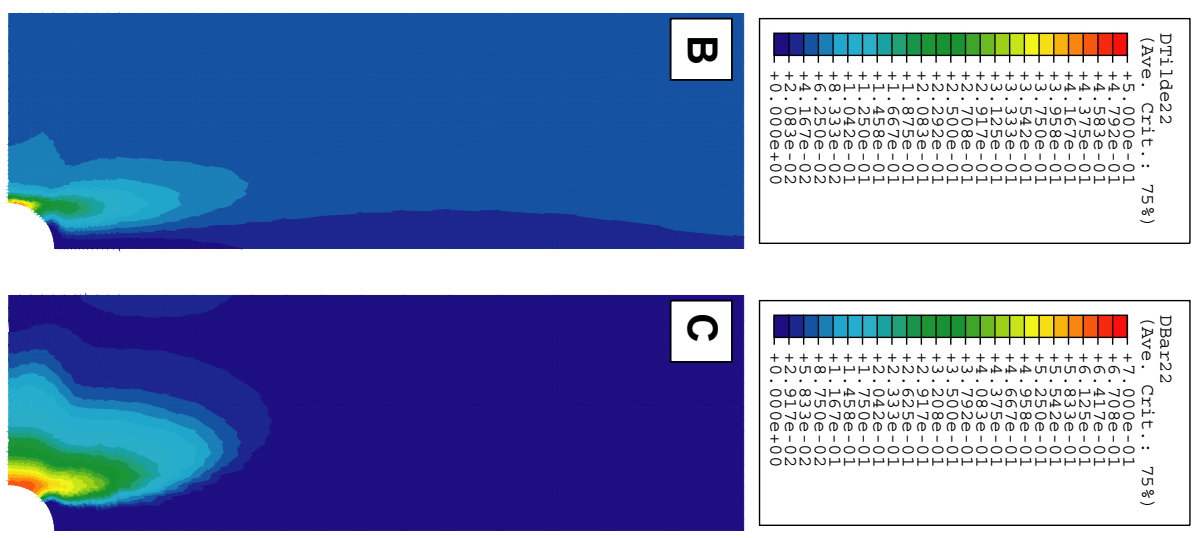

Fig. 2. Map of damage in the $90^{\circ}$ ply: (A) damage along the fibers $d_{\mathrm{F}}$, (B) diffuse damage $\tilde{d}^{\prime}$ and (C) microcracking damage $\bar{d}_{22}$. Longitudinal deformation of the sample: $0.633 \%$.
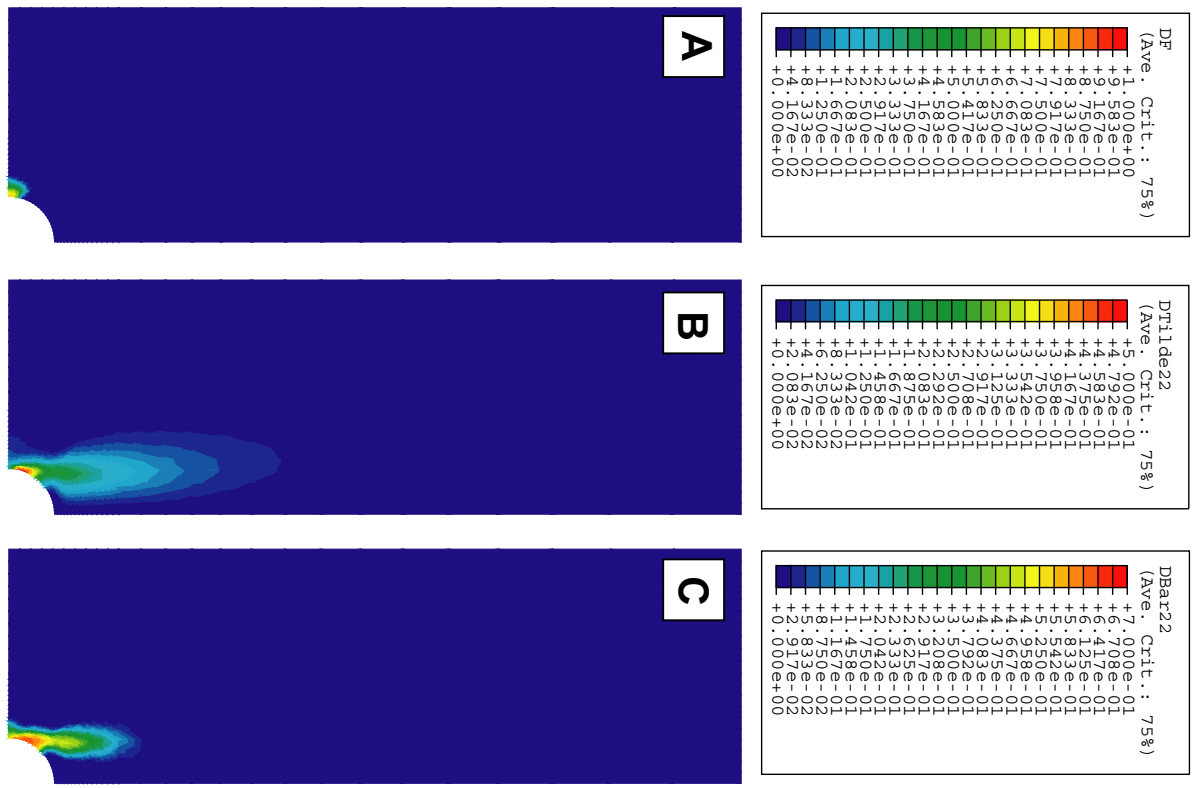

Fig. 3. Map of damage in the $0^{\circ}$ ply: (A) damage along the fibers $d_{\mathrm{F}}$, (B) diffuse damage $\tilde{d}^{\prime}$, and (C) microcracking damage $\bar{d}_{22}$. Longitudinal deformation of the sample: $0.633 \%$.

distribution of this damage according to the underlying degradation mechanisms.

\section{Conclusion}

We introduced an intralaminar model formulated within the framework of damage mechanics and based on a micromechanics reference model. This strategy enables one to take advantage of recent advances in commercial analysis codes, which we illustrated with a simple example.
Future works will focus on the extension of this approach to the interlaminar interface and the corresponding implementation. Indeed, the homogenization method proposed in [24] enables one to couple the intra and interlaminar degradations explicitly in the framework of a nonlocal damageable interface model. Therefore, future works will concentrate on the implementation of this model in order to improve, most of all, the simulation of the coupling between transverse cracking and delamination, which is a major industrial problem. 
Annex A. Let us consider a stratified medium containing a layer of interest $(S)$, initially transverse isotropic, the rest of the stack being represented in homogeneous fashion through peripheral parts $(S)$ and $\left(S^{\prime \prime}\right)$. We are studying a periodical cell of length $l$ of this stack.

This cell, initially healthy, is subjected to a uniform macroscopic loading characterized by the plane part of the macroscopic strain $\left[\varepsilon_{11}, \varepsilon_{22}, \varepsilon_{12}\right]$ and by the out-of-plane part of the stresses $\left[\sigma_{13}, \sigma_{23}, \sigma_{33}\right]$. This choice for the description of the macroscopic loading is not inconsequential and results from the micro-meso relations developed in [11]. Then, generally speaking, the stress state in the healthy cell under such loading is denoted $\tilde{\sigma}$ and called "effective stress".

Now, let us assume that layer $(S)$ has been degraded due to a transverse cracking mechanism of density $\rho=H / l$. In order to find the solution of this new problem, the "healthy" solution $\tilde{\sigma}$ must be corrected through three periodic residual problems corresponding to the three residuals $\left[-\tilde{\sigma}_{22}\right] \cdot \underline{n}_{2},\left[-\tilde{\sigma}_{12}\right] \cdot \underline{n}_{2}$ and $\left[-\tilde{\sigma}_{23}\right] \cdot \underline{n}_{2}$ along the lips of the transverse cracks. This layer homogenization problem has some remarkable properties and leads to the definition of three damage indicators $\bar{d}_{22}, \bar{d}_{12}$ and $\bar{d}_{23}$ on the energy level, such that the strain energy of the degraded layer can be expressed as $[10,11]$ :

$$
\begin{aligned}
2 e_{d}= & {\left[\begin{array}{c}
\tilde{\sigma}_{11} \\
\tilde{\sigma}_{22} \\
\tilde{\sigma}_{33}
\end{array}\right]^{\mathrm{t}}\left[\begin{array}{ccc}
\frac{1}{E_{1}^{0}} & -\frac{v_{12}^{0}}{E_{1}^{0}} & -\frac{v_{12}^{0}}{E_{1}^{0}} \\
-\frac{v_{12}^{0}}{E_{1}^{0}} & \frac{1-\left[\tilde{\sigma}_{22}+\bar{d}_{22}\right.}{E_{2}^{0}} & -\frac{v_{23}^{0}}{E_{2}^{0}} \\
-\frac{v_{12}^{0}}{E_{1}^{0}} & -\frac{v_{23}^{0}}{E_{2}^{0}} & \frac{1}{E_{2}^{0}}
\end{array}\right]\left[\begin{array}{c}
\tilde{\sigma}_{11} \\
\tilde{\sigma}_{22} \\
\tilde{\sigma}_{33}
\end{array}\right] } \\
& +\frac{\left(1-\bar{d}_{12}\right) \tilde{\sigma}_{12}^{2}}{2 G_{12}^{0}}+\frac{\tilde{\sigma}_{13}^{2}}{2 G_{12}^{0}}+\frac{\left(1-\bar{d}_{23}\right) \tilde{\sigma}_{23}^{2}}{2 G_{23}^{0}}
\end{aligned}
$$

Now, in order to obtain a more standard expression, let us substitute the actual stress for the effective stress in Eq. (22). These stresses are related through

$$
\begin{aligned}
& \sigma_{11}=\tilde{\sigma}_{11}-\frac{v_{12}^{0} \bar{d}_{22}}{1-v_{12}^{0} v_{21}^{0}} \tilde{\sigma}_{22}\left[\tilde{\sigma}_{22}\right]^{+} \\
& \sigma_{22}=\tilde{\sigma}_{22}-\frac{\bar{d}_{22}}{1-v_{12}^{0} v_{21}^{0}} \tilde{\sigma}_{22}\left[\tilde{\sigma}_{22}\right]^{+} \\
& \sigma_{33}=\tilde{\sigma}_{33} \\
& \sqrt{2} \sigma_{12}=\sqrt{2}\left(1-\bar{d}_{12}\right) \tilde{\sigma}_{12} \\
& \sqrt{2} \sigma_{13}=\sqrt{2} \tilde{\sigma}_{13} \\
& \sqrt{2} \sigma_{23}=\sqrt{2}\left(1-\bar{d}_{23}\right) \tilde{\sigma}_{23}
\end{aligned}
$$

In addition, one has

$$
\left[\tilde{\sigma}_{22}\right]^{+}=\left[\sigma_{22}\right]^{+}
$$

The transformation of Eq. (22) yields the equation in actual stresses

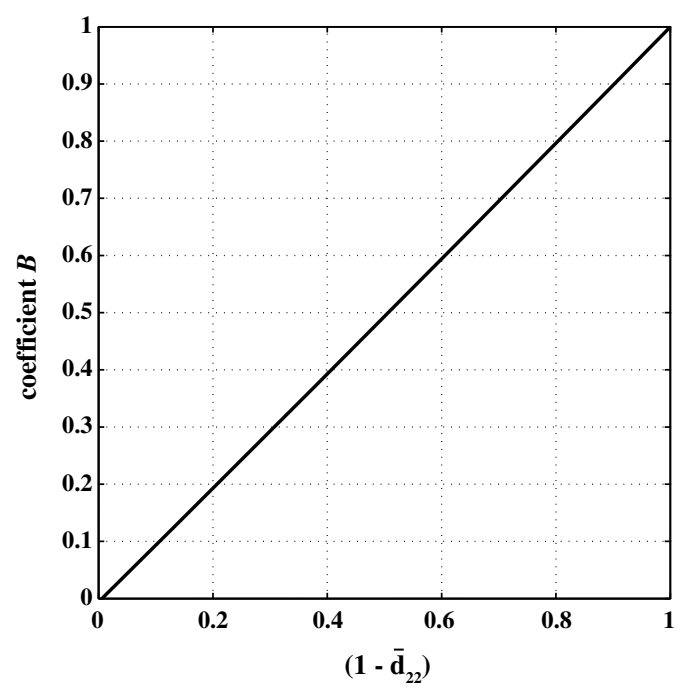

Fig. 4. Plot of coefficient B (using classical properties for carbon-epoxy).

$$
\begin{aligned}
2 e_{d}= & {\left[\begin{array}{l}
\sigma_{11} \\
\sigma_{22} \\
\sigma_{33}
\end{array}\right]^{\mathrm{t}}\left[\begin{array}{ccc}
\frac{1}{E_{1}^{0}} & -\frac{v_{12}^{0}}{E_{1}^{0}} & -\frac{v_{12}^{0}}{E_{1}^{0}} \\
-\frac{v_{12}^{0}}{E_{1}^{0}} & \frac{1}{\mathscr{A} E_{2}^{0}} & -\frac{v_{23}^{0}}{\mathscr{P E} E_{2}^{0}} \\
-\frac{v_{12}^{0}}{E_{1}^{0}} & -\frac{v_{23}^{0}}{\mathscr{B} E_{2}^{0}} & \frac{1}{E_{2}^{0}}
\end{array}\right]\left[\begin{array}{l}
\sigma_{11} \\
\sigma_{22} \\
\sigma_{33}
\end{array}\right] } \\
& +\frac{\sigma_{12}^{2}}{2 G_{12}^{0}\left(1-\bar{d}_{12}\right)}+\frac{\sigma_{13}^{2}}{2 G_{12}^{0}}+\frac{\sigma_{23}^{2}}{2 G_{23}^{0}\left(1-\bar{d}_{23}\right)}
\end{aligned}
$$

with

$$
\begin{aligned}
& \mathscr{A}=\frac{\left(\left(E_{1}^{0}-v_{12}^{0^{2}} E_{2}^{0}\right)-\left[\sigma_{22}\right]^{+} \bar{d}_{22} E_{1}^{0}\right)}{\left(E_{1}^{0}-v_{12}^{0^{2}} E_{2}^{0}\right)-\left[\sigma_{22}\right]^{+} \bar{d}_{22} v_{12}^{0^{2}} E_{2}^{0}} \\
& \mathscr{B}=\frac{\left(\left(E_{1}^{0}-v_{12}^{0^{0}} E_{2}^{0}\right)-\left[\sigma_{22}\right]^{+} \bar{d}_{22} E_{1}^{0}\right)}{\left(\left(E_{1}^{0}-v_{12}^{0^{2}} E_{2}^{0}\right)+\left[\sigma_{22}\right]^{+} \bar{d}_{22} \frac{0_{12}^{0} v_{23}^{0}}{v_{23}^{0}}\right)}
\end{aligned}
$$

We had already proven [15] that

$\mathscr{A} \approx\left(1-\left[\sigma_{22}\right]^{+} \bar{d}_{22}\right)$

We also have, with an excellent approximation (Fig. 4)

$\mathscr{B} \approx\left(1-\left[\sigma_{22}\right]^{+} \bar{d}_{22}\right)$

which leads to the expression of Eq. (4).

\section{References}

[1] N. Laws, G. Dvorak, Journal of Composite Materials 22 (1988) 900 916.

[2] J. Nairn, S. Hu, International Journal of Fracture 57 (1992) 1-24.

[3] G. Dvorak, N. Laws, Journal of Composite Materials 21 (1987) 309329.

[4] L. Boniface, P. Smith, S. Ogin, M. Bader, In: Proceedings of the 6th International Conference on Composite Materials, vol. 3, 1987, pp. $156-165$.

[5] S.-H. Lim, S. Li, Composites Part A 36 (2005) 1467-1476.

[6] P. Ladevèze, Sur la mécanique de l'endommagement des composites, in: C. Bathias, D. Menkès (Eds.), Comptes-rendus des JNC5, Pluralis Publication, Paris, 1986, pp. 667-683. 
[7] P. Ladevèze, About a damage mechanics approach, in: D. Baptiste (Ed.), Mechanics and Mechanisms of Damage in Composite and Multimaterials, MEP, 1989, pp. 119-142.

[8] P. Ladevèze, E. LeDantec, Composite Science and Technology 43 (3) (1992) 257-267.

[9] O. Allix, P. Ladevèze, Composite Structures 22 (1992) 235-242.

[10] P. Ladevèze, G. Lubineau, Composite Science and Technology 61 (15) (2001) 2149-2158.

[11] P. Ladevèze, G. Lubineau, CR Mecanique 331 (2003) 537-544, in French.

[12] P. Ladevèze, International Congress of Theoretical and Applied Mechanics XXI, 2004.

[13] P. Ladevèze, G. Lubineau, D. Violeau, International Journal of Fracture 137 (1-4) (2006) 139-150.

[14] Z. Hashin, Mechanical of Materials 4 (1985) 121-136.

[15] P. Ladevèze, G. Lubineau, Mechanics of Materials 35 (2003) 763-776.

[16] P. Ladevèze, Multiscale computational damage modelling of laminate composites, Course CISM 2005.

[17] J. Nairn, Matrix microcracking in composites, in: R. Talreja, J.-A.E. Manson (Eds.), Polymer Matrix Composites, Comprehensive Composite Materials, vol. 2, Elsevier Science, 2000, pp. 403-432.

[18] J. Berthelot, Applied Mechanics Reviews 56 (1) (2003) 1-37.

[19] E. Gamstedt, B. Sjrögren, Composites Science and Technology (59) (1999) $167-178$.
[20] B. Sjögren, L. Berglund, Composites Science and Technology (60) (2000) 9-21.

[21] F. Lagattu, M. Lafarie-Frenot, Composites Science and Technology (60) (2000) 605-612.

[22] S. Ogihara, N. Takeda, Composite Science and Technology 54 (4) (1995) 395-404

[23] P. LadevFze, A. Nouy, Computer Methods in Applied Mechanics 192 (2003) 3061-3087.

[24] P. Ladevèze, G. Lubineau, D. Marsal, Composite Science and Technology 66 (6) (2005) 698-712.

[25] A. Caporale, R. Luciano, E. Sacco, Computers and Structures Available online.

[26] C. Henaff-Gardin, M. Lafarie-Frenot, J. Brillaud, A.E. Mahi, Influence of the stacking sequence on fatigue transverse ply cracking in cross-ply composite laminates, in: J. Masters (Ed.), Damage detection in composite materials, ASTM-STP 1128, 1992, pp. 236255.

[27] P. LadevFze, Computers and Structures 44 (1992) 79-87.

[28] O. Allix, J. Deu, Engineering Transactions - Polish Academy of Science - Institute of Fundamental Technological Research 45 (1) (1997) 29-46.

[29] S. Yashiro, T. Okabe, N. Toyama, N. Takeda, International Journal of Solids and Structures 44 (2007) 603-613. 